\title{
Characterization of the hot pepper (Capsicum frutescens) fruit ripening regulated by ethylene and $A B A$
}

\author{
Bing-Zhu Hou, Chun-Li Li, Ying-Yan Han and Yuan-Yue Shen * (D)
}

\begin{abstract}
Background: Ripening of fleshy fruits has been classically defined as climacteric or non-climacteric. Both types of ripening are controlled by plant hormones, notably by ethylene in climacteric ripening and by abscisic acid (ABA) in non-climacteric ripening. In pepper (Capsicum), fruit ripening has been widely classified as non-climacteric, but the ripening of the hot pepper fruit appears to be climacteric. To date, how to regulate the hot pepper fruit ripening through ethylene and ABA remains unclear.

Results: Here, we examined ripening of the hot pepper (Capsicum frutescens) fruit during large green (LG), initial colouring (IC), brown (Br), and full red (FR) stages. We found a peak of ethylene emission at the IC stage, followed by a peak respiratory quotient at the $\mathrm{Br}$ stage. By contrast, $\mathrm{ABA}$ levels increased slowly before the $\mathrm{Br}$ stage, then increased sharply and reached a maximum level at the FR stage. Exogenous ethylene promoted colouration, but exogenous ABA did not. Unexpectedly, fluridone, an inhibitor of ABA biosynthesis, promoted colouration. RNAsequencing data obtained from the four stages around ripening showed that ACO3 and NCED1/3 gene expression determined ethylene and ABA levels, respectively. Downregulation of ACO3 and NCED1/3 expression by virusinduced gene silencing (VIGS) inhibited and promoted colouration, respectively, as evidenced by changes in carotenoid, ABA, and ethylene levels, as well as carotenoid biosynthesis-related gene expression. Importantly, the retarded colouration in ACO3-VIGS fruits was rescued by exogenous ethylene.
\end{abstract}

Conclusions: Ethylene positively regulates the hot pepper fruit colouration, while inhibition of ABA biosynthesis promotes colouration, suggesting a role of ABA in de-greening. Our findings provide new insights into processes of fleshy fruit ripening regulated by $A B A$ and ethylene, focusing on ethylene in carotenoid biosynthesis and $A B A$ in chlorophyll degradation.

Keywords: Hot pepper (Capsicum frutescens), Fruit ripening, Carotenoid biosynthesis, Chlorophyll degradation, Ethylene, Abscisic acid

\section{Background}

Peppers (Capsicum), as the most widely cultivated vegetable crop, play important roles in human food, nutrition, and health [1]. The Capsicum genus includes five species: C. annuum, C. chinense, C. frutescens, C. pubescens, and C. baccatum, which are known as bell, chili, jalapeno, cayenne, and cherry types, respectively [2-4]. Generally, bell peppers (C. апnиит) are harvested at the horticulturally mature green stage for fresh food, while

\footnotetext{
* Correspondence: sfmn@tom.com

College of Plant Science and Technology, Beijing Key Laboratory for

Agricultural Application and New Technique, Beijing University of Agriculture, Beijing 102206, China
}

hot peppers (C. frutescens) are harvested at the ripening stage for processing or drying. The pepper fruit ripening has been typically classified as non-climacteric [5-7]. However, given the wide diversity of peppers, patterns of ethylene production and respiratory rates vary with pepper species, and the hot pepper fruit ripening appears to be climacteric [8]. To our knowledge, how to regulate the hot pepper fruit ripening by ethylene and ABA remains unclear.

Ripening of fleshy fruits is typically classified as climacteric or non-climacteric based on ethylene emission and respiration during ripening [9-11]. During ripening, climacteric fruits show a burst in ethylene emission and

(c) The Author(s). 2018 Open Access This article is distributed under the terms of the Creative Commons Attribution 4.0 International License (http://creativecommons.org/licenses/by/4.0/), which permits unrestricted use, distribution, and 
respiration, whereas no peak in ethylene emission occurs in non-climacteric fruits $[11,12]$. Based on various tomato (Solanum lycopersicum) mutants, including never-ripe $(\mathrm{nr})$, colourless non-ripening (cnr), ripening inhibitor (rin), and non-ripening (nor) mutants, the molecular mechanisms of climacteric fruit ripening have been defined by ethylene biosynthesis and signalling $[13,14]$. Ethylene biosynthesis is controlled by two key steps which are catalysed sequentially by 1-aminocyclopropane-1-carboxylic acid (ACC) synthases (ACSs) and ACC oxidases (ACOs) [15]. Ethylene is perceived by a family of five ethylene receptors (ETR1, ETR2, ERS1, ERS2, and EIN4) which interact with a downstream constitutive triple response 1 (CTR1) kinase, which activates ethylene insensitive 2 (EIN2) by a negative mechanism and in turn evokes a transcriptional cascade involving EIN3/ EIN3-like (EIL) and ethylene responsive factors [16-20]. A coordinated regulation between ethylene biosynthesis and ethylene signalling by the tomato MADS-box protein, LeMADS-RIN, is required for ripening [21].

Although the mechanisms of non-climacteric fruit ripening regulated by plant hormones are not fully understood [14, 19], increasing evidence indicates an important role of ABA in ripening [22-29]. Tobacco rattle virus (TRV)-induced gene silencing (VIGS) in strawberry fruit, a model of non-climacteric ripening, showed that downregulation of the mRNA expression levels of FaNCED1 or FaBG3, key to ABA biosynthesis, led to a significant reduction in $\mathrm{ABA}$ contents and then inhibition of ripening, respectively [23, 24]. Similarly, downregulation of the mRNA expression levels of FaPYR1/ $F a A B A R$ and FaABI1/FaSnRK2.6, key to ABA signalling, affected ripening [22, 23, 27]. FaMYB10 is a signal transduction mediator to play a role in ABAR (putative ABA receptor)-mediated anthocyanin synthesis during strawberry fruit ripening [28]. Vital roles for hormones in fleshy fruit ripening are now accepted, particularly for ethylene in climacteric fruit and for ABA in non-climacteric fruit $[12,14,19,20,30,31]$.

Notably, the ripening of some non-climacteric fruits in guava, melon, Japanese plum, Asian pear, and hot pepper, is climacteric $[32,33]$. Pepper fruit ripening is typically considered to be non-climacteric [5-7, 34-38], while some hot pepper fruit ripening is climacteric, and patterns of ethylene production and respiratory rates differ with pepper species $[8,39]$. Thus, characterisation of hot pepper fruit ripening regulated by $\mathrm{ABA}$ and ethylene contributes to understanding of fleshy fruit ripening.

In this study, we used a red, clustered, upright, bullet-shaped and hot pepper (Capsicum frutescens cv. 'Chaotianjiao 6') to examine fruit ripening during large green (LG), initial colouring (IC), brown (Br), and full red (FR) stages. We performed a series of physiological, pharmacological [ethylene and its inhibitor 1-methylcyclopropene
(1-MCP), and ABA and its inhibitor, fluridone], RNA-sequencing (RNA-seq), and VIGS experiments to assess the roles of ethylene and ABA in the hot pepper fruit ripening. We found that $\mathrm{ABA}$ and ethylene regulate hot pepper ripening differently, showing either climacteric or non-climacteric characteristics.

\section{Results \\ Morphological and physiological changes at the onset of pepper fruit ripening}

We divided the development of 'Chaotianjiao 6' (Additional file 1: Figure S1) pepper fruit into six stages: young green (YG), small green (SG), large green (LG), initial colouring (IC), brown (Br), and full red (FR) at 1, 2, 3, 4, 5, and 6 weeks after anthesis, respectively, of which the later four stages corresponded to the onset of ripening (Fig. 1a). Concurrent with the onset of ripening, glucose, fructose, and carotenoid contents increased rapidly (Fig. 1b, d), while sucrose and starch contents declined sharply after the IC stage (Fig. 1b, c) and chlorophyll contents decreased gradually (Fig. 1d). Notably, ethylene emission reached a peak at the IC stage, concurrent with changes in colouration (Fig. 1f), followed by a peak in the respiratory quotient (RQ) at the $\mathrm{Br}$ stage (Fig. 1e). Interestingly, the drastic reduction in the RQ (Fig. 1e) was accompanied by rapid accumulation of ABA after the $\mathrm{Br}$ stage (Fig. 1g). These results suggested that both ethylene and ABA might be involved in ripening.

\section{Effects of $A B A$ and ethylene and their inhibitors on pepper fruit ripening}

It is previously reported that detached pepper fruits are suitable for the study of ripening [8]. Therefore, we used detached LG-stage pepper fruits to investigate the effects of ABA and ethylene on ripening. LG fruits were treated for 8 days with $50 \mu \mathrm{M}$ ABA, $100 \mu \mathrm{M}$ fluridone, $50 \mu \mathrm{M}$ ethephon, or $1 \mathrm{~mL} / \mathrm{L}$ 1 -MCP ( $n=7$, three replications). Relative to the control treatment with water, colouration was promoted by ethephon and fluridone (21 FR fruits and 21 orange fruits, respectively), whereas the $\mathrm{ABA}$ and 1-MCP treatments had no effect on colouration (Fig. 2a). Ethylene emission was significantly promoted and inhibited by the ethephon and 1-MCP treatments, respectively, while was not affected significantly by the ABA and fluridone treatments (Fig. 2b). However, the endogenous ABA levels were significantly affected by the ABA and fluridone treatments, resulting in endogenous ABA levels of $207 \pm 12$ and $34 \pm 2.1 \mu \mathrm{g} / \mathrm{g}$ fresh weight (FW), respectively. These results suggest that ethylene plays a role in the regulation of ripening, and that the role of $\mathrm{ABA}$ is complex. 


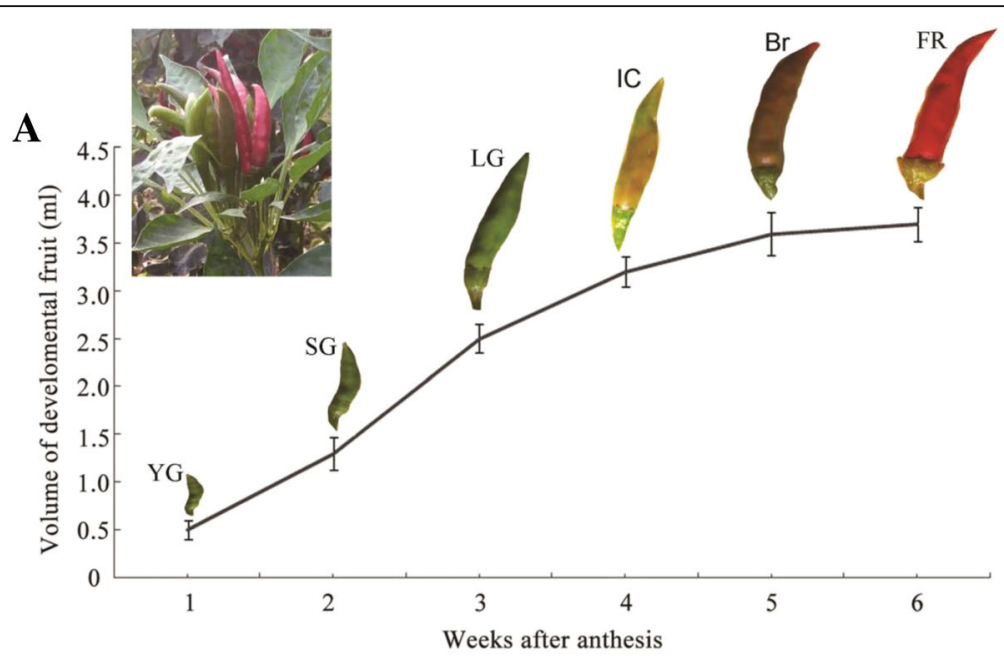

B

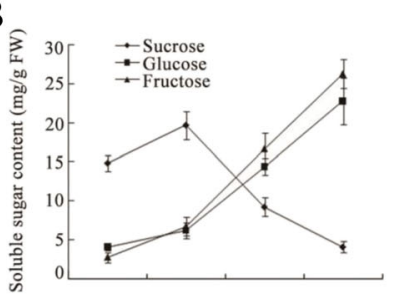

D

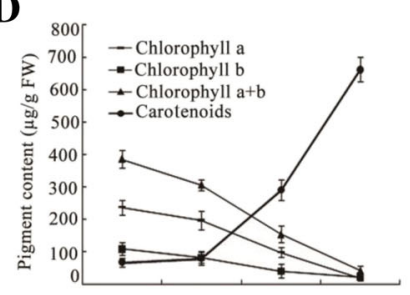

F

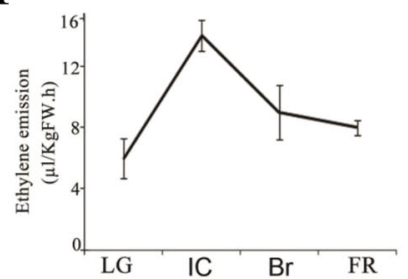

C

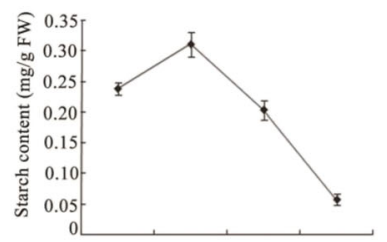

$\mathbf{E}$

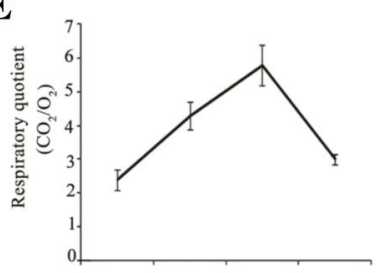

G

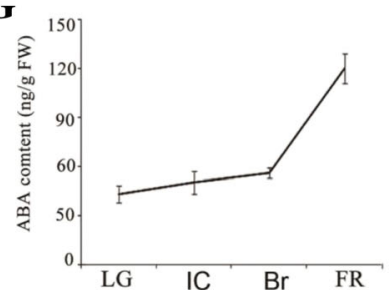

Fig. 1 Morphological and physiological variation in pericarp tissues during pepper fruit ripening. The developmental pepper fruit was divided into six stages - young green (YG), small green (SG), large green (LG), initial colouring (IC), brown (Br), and full red (FR) - at 1, 2, 3, 4, 5, and 6 weeks after fruit set, respectively. Pericarp tissues were used for physiological analysis. Changes in (a) size and colour during fruit development, (b) soluble sugar (sucrose, glucose, and fructose) content, (c) starch content, (d) pigment content, (e) respiratory quotient, (f) ethylene emission, and (g) ABA content. Error bars represent the standard error (SE, $n=3)$

\section{RNA-seq analysis of gene expression during the onset of pepper fruit ripening}

To understand the molecular mechanisms by which plant hormones regulate pepper fruit ripening, we performed RNA-seq analysis of cDNA libraries corresponding to four stages during the onset of pepper fruit ripening. Genetic databases used to annotate unigenes identified by RNA-seq analysis including the National
Center for Biotechnology Information non-redundant protein database, SWISS-PROT, TrEMBL, Cdd, Pfam, the KOG database, the Kyoto Encyclopedia of Genes and Genomes, and Gene Ontology based on an E-value of $1 \mathrm{e}^{-5}$ and identity of $30 \%$. Differentially expressed genes (DEGs) were identified between the LG and IC stages as 2337 genes with 1145 upregulated and 1192 downregulated, the LG and Br stages as 3018 genes with 


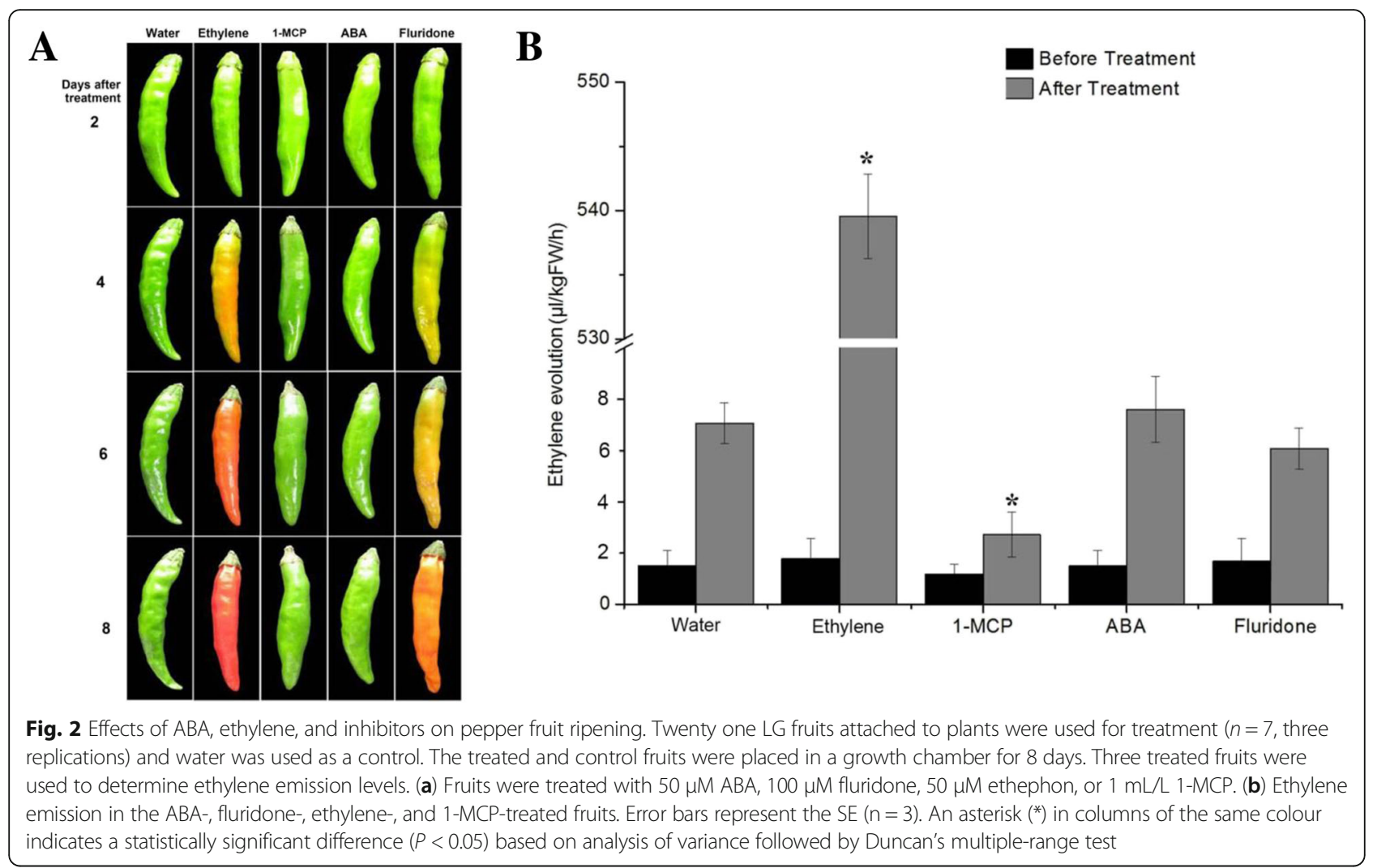

1482 upregulated and 1536 downregulated, the LG and FR stages as 3801 genes with 1522 upregulated and 2279 downregulated, the IC and $\mathrm{Br}$ stages as 628 genes with 317 upregulated and 311 downregulated, the IC and FR stages as 1858 genes with 686 upregulated and 1172 downregulated, and the $\mathrm{Br}$ and FR stages as 1354 genes with 524 upregulated and 830 downregulated (Fig. 3a). Some of these DEGS were specific to particular pairs of ripening stages; 237 DEGs were specific to LG/IC, 417 DEGs were specific to LG/Br, 1464 DEGs were specific to LG/FR (Fig. 3b), 189 DEGs were specific to $\mathrm{IC} / \mathrm{Br}$, and 1419 DEGs were specific to IC/FR (Fig. 3c). Among these DEGs, we identified genes related to phytohormone and carotenoid pathways, including carotenoid biosynthesis genes (PSY/crtB, PDS/crtP, ZEP, ZDS/crtQ, $B C H / c r t Z 1$, and $C C S / l c y B / \operatorname{crt} Y)$, ABA biosynthesis and metabolism genes (ABA1/ZEP, NCED1, NCED3, AAO1, and CYP707A1), ABA signalling genes (PYL12, PP2C37, PP2C37-like, SnRK2, and ABI5), ethylene biosynthesis and signalling genes (ACO3, CTR1, CTR2, EIN2, EIN3, and ERF1), auxin biosynthesis and signalling genes ( $G H 3$ and $I A A$ ), a GA (gibberelin) signalling gene (GID1), a JA (jasmonic acid) signalling gene (MYC2), and a cytokinin signalling gene (ARR5; Fig. 4).

Consistent with the changes in fruit colouration during ripening, the DEGs with continuously decreasing transcript levels included SnRK2 and GID1, and the DEGs with continuously increasing transcripts included EIN3, ERF2, ABI5, PP2C37, NCED1, CYP707A1, AAO, GH3, and MYC2. The DEGs with highest transcript levels at the IC stage included EIN2, ACO3, CTR1, IAA1, and ARR5, and the DEGs with highest transcript levels at the $\mathrm{Br}$ stage included CTR2, NCED3, ZDS, PDS, crtB, crtZ1, and ZEP (Fig. 4). Importantly, ABA-related genes $(n=10)$ constituted the largest portion of the phytohormone-related DEGs during ripening, followed by ethylene-related genes $(n=6)$, and only two DEGs related to other phytohormones were identified. Interestingly, transcription of the ethylene-related DEGs during ripening showed a coordinated expression pattern between ethylene biosynthesis and signalling, whereas transcripts of the ABA-related DEGs showed no coordinated expression patterns between ABA biosynthesis and signalling (Fig. 4). More importantly, ACO3 transcript levels exhibited a trend similar to ethylene levels, whereas NCED1 and NCED3 transcript levels, in particular NCED1, exhibited trends consistent with $\mathrm{ABA}$ contents (Figs. 1, 4). Taken together, the transcriptome data related to phytohormone-pathway DEGs suggest that ethylene plays an important role in ripening, and that the role of ABA is complex.

\section{VIGS of key genes to ABA and ethylene biosynthesis during pepper fruit ripening}

To confirm the roles of ACO3, NCED1, and NCED3 in ABA and ethylene biosynthesis, we examined the 


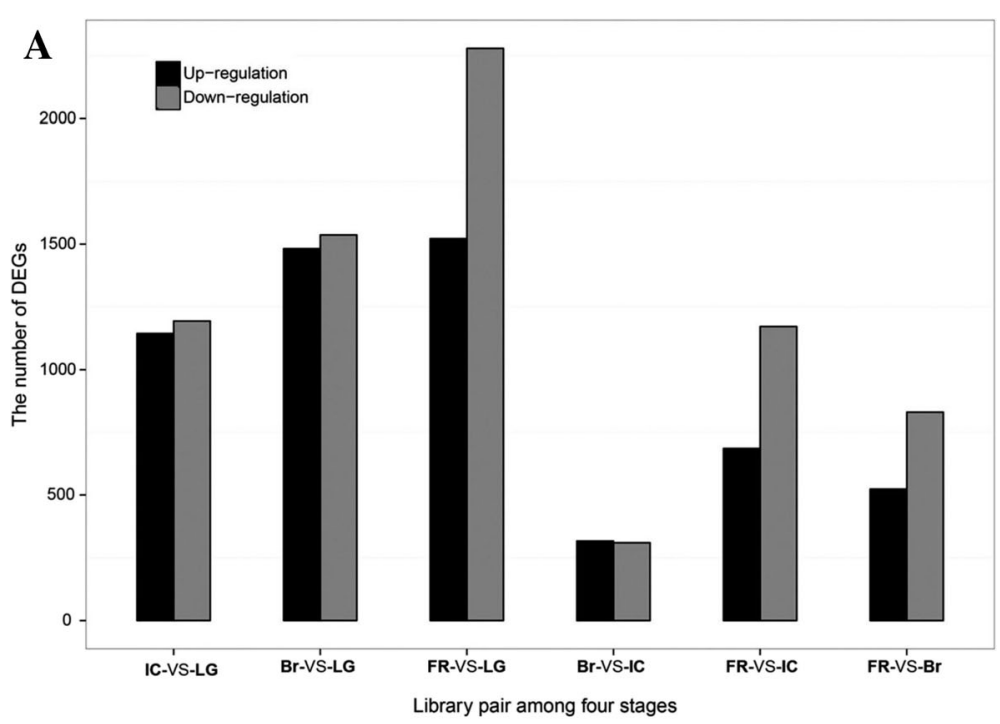

B

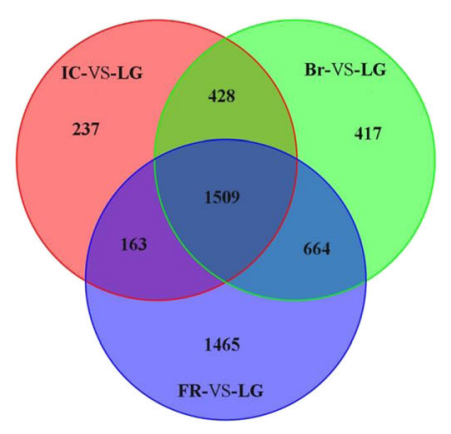

C

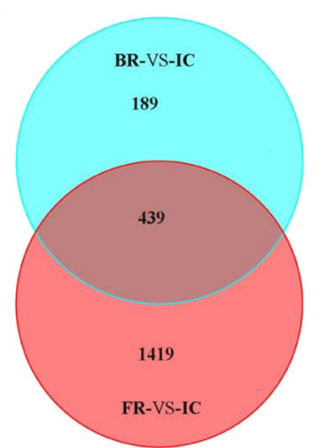

Fig. 3 Differentially expressed genes (DEGs) between each pair of ripening stage CDNA libraries. Four CDNA libraries corresponded to the LG, IC, $\mathrm{Br}$, and FR fruit ripening stages. (a) Numbers of up- and down-regulated DEGs between library pairs. (b) Venn diagram comparing the DEGs between the IC, Br, and FR libraries and the LG library. (c) Venn diagram comparing the DEGs between the Br and FR libraries and the IC library

RNA-seq data for the presence of transcripts of the $A C O$ and NCED gene families at the onset of ripening. We found that of the four $A C O$ gene family members, $A C O 3$ might play a key role in ethylene biosynthesis (Fig. 5a), and that of the three NCED gene family members, NCED1 and NCED3 might be important in ABA biosynthesis (Fig. 5b).

To determine the roles of $\mathrm{ABA}$ and ethylene in pepper fruit ripening, we used TRV-mediated VIGS [40] to downregulate the mRNA expression levels of $A C O 3$, NCED1, and NCED3 during pepper fruit ripening. A mixture of Agrobacterium strain GV3101 cultures containing the pTRV1 vector and the PTRV2 vector carrying a 567-bp ACO3, a 535-bp NCED1, or a 602-bp NCED3 gene fragment in a 1:1 ratio were syringe-infiltrated into LG fruits attached to the plants ( $n=7$, three replications), and the control fruits were infiltrated with TRV vectors alone (Fig. 5c). Six days after infiltration, most NCED1/3-VIGS fruits had initiated colouration (18 IC fruits, 3 LG fruits), while all control fruits remained light-green in colour (21 LG fruits; Fig. 5d), demonstrating that the downregulation of $N C E D 1 / 3$ expression promoted fruit colouration. In contrast, 3 weeks after infiltration, all control fruits infiltrated with empty TRV vectors had turned fully red, while the inoculated ACO3-VIGS fruits exhibited chimeric phenotypes (21 chimeras; Fig. 5e), demonstrating that the downregulation of $A C O 3$ expression inhibited fruit colouration. Importantly, these $A C O 3$-VIGS chimeric fruits were rescued by exogenous application of $50 \mu \mathrm{M}$ ethephon 5 days after treatment (Fig. 5f).

To confirm suppression of ACO3 and NCED1/3 gene expression by VIGS at molecular levels, we performed quantitative polymerase chain reaction (qPCR) analysis. The ACO3 mRNA level was downregulated in VIGS fruits more than $80 \%$ relative to the control, and the average mRNA levels of NCED1 and NCED3 in VIGS fruits were downregulated more than $70 \%$ relative to the control (Fig. 5g). The combination of transgenic phenotypes and molecular analysis demonstrated that ACO3 


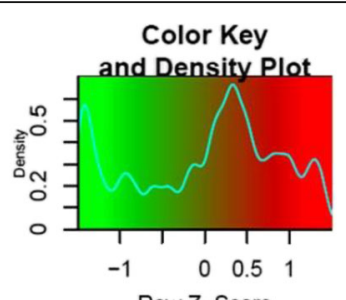

LG $\quad$ IC $\quad \mathrm{Br} \quad \mathrm{FR}$

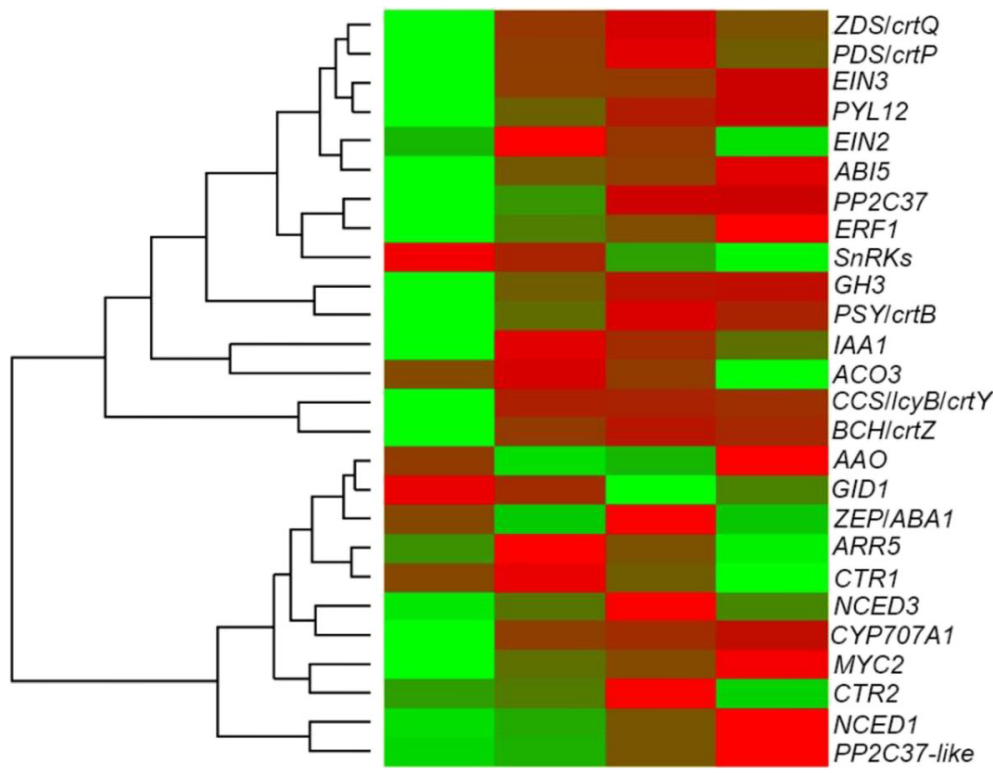

Fig. 4 Heat map and cluster analysis of transcripts of genes related to ripening. Gene expression level data were normalised to $z$-scores using the formula $\log _{10}(F P K M+1)$ by colour key and density plot. The transition from green to red represents the value of gene expression from low to high during fruit ripening. The genes annotated in relevant pathways: carotenoid biosynthesis (PSY/crtB: 15-cis-phytoene synthase; PDS/crtP: 15cis-phytoene desaturase; ZDS/crtQ: zeta-carotene desaturase; BCH/crtZ1: beta-carotene hydroxylase 1; CCS/lcyB/crtY: capsanthin/capsorubin synthase); ABA biosynthesis and metabolism (ZEP/ ABA1: zeaxanthin epoxidase; NCED1: 9-cis-epoxycarotenoid dioxygenase 1; NCED3: 9-cisepoxycarotenoid dioxygenase 3; AAO1: abscisic-aldehyde oxidase 1; CYP707A1, abscisic acid 8'-hydroxylase); ABA signaling (PYL12: abscisic acid receptor; PP2C37: protein phosphatase 2C 37; PP2C37-like: protein phosphatase 2C 37-like; SnRK2: serine/threonine-protein kinase SRK2-like; ABI5: abscisic acid-insensitive 5-like protein); ethylene biosynthesis and signaling (ACO3: 1-aminocyclopropane-1-carboxylate oxidase 3; CTR1: ethylene response sensor 1; CTR2: ethylene response sensor 2; EIN2: ethylene-insensitive protein 2; EIN3: ethylene-insensitive protein 3; ERF1: ethyleneresponsive transcription factor 1B-like); IAA biosynthesis and signaling (GH3: indole-3-acetic acid-amido synthetase GH3.1; IAA, auxin-responsive protein IAA); GA signaling (GID1, gibberellin receptor GID1); JA signaling (MYC2, transcription factor MYC2); cytokinin signaling (ARR5: twocomponent response regulator 5)

positively regulates colouration, whereas NCED1/3 negatively regulates colouration.

\section{Characterisation of colouration in transgenic pepper fruits at the physiological and molecular levels}

To understand colouration in the transgenic pepper fruits, we examined a set of physiological and molecular parameters in VIGS fruits in which NCED (mixed samples) and $A C O 3$ transcripts were downregulated more than 70 and $80 \%$, respectively, relative to their corresponding controls. The measured parameters included ethylene emission, ABA content, pigment (chlorophyll, ß-carotene, zeaxanthin, and capsanthin) concentrations, and transcript levels of carotenoid biosynthesis-related DEGs (PSY, PDS, ZDS, ZEP, BCH, and CCS). ABA content and ethylene emission were significantly reduced in the NCEDs-VIGS and ACO3-VIGS fruits, respectively (Fig. 6a, b). The downregulation of NCED expression promoted capsanthin, zeaxanthin, and $ß$-carotene accumulation, but decreased the chlorophyll content (Fig. 6c), finally accelerated colouration. In contrast, the downregulation of $A C O 3$ expression inhibited capsanthin, zeaxanthin, and $ß$-carotene accumulation as well as chlorophyll degradation (Fig. 6d), which retarded colouration. The promotion and inhibition of colouration in the NCEDs-VIGS and ACO3-VIGS fruits, respectively, were consistent with the changes in transcript levels of carotenoid biosynthesis-related genes, such as PSY, PDS, ZDS, ZEP, BCH, and CCS (Fig. 6e, f). Our results demonstrated that the downregulation of NCED expression inhibited ABA synthesis, finally promoted carotenoid biosynthesis-related 
A

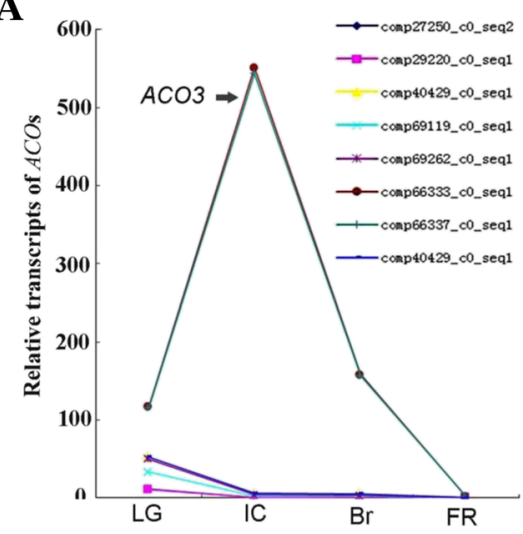

C

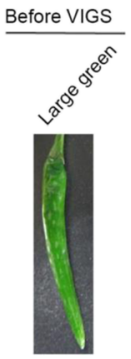

G
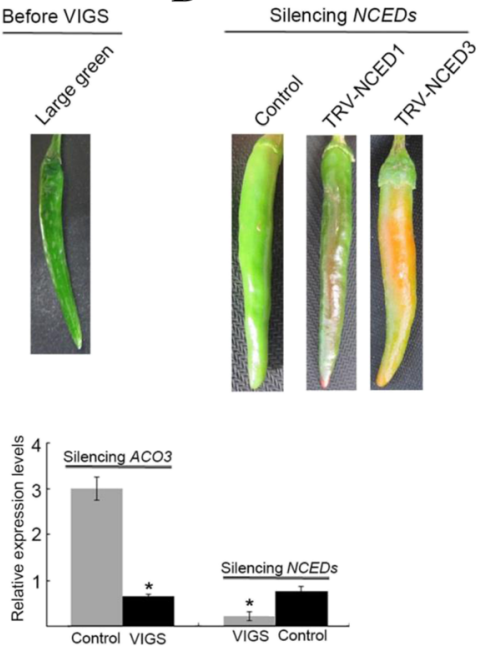

B

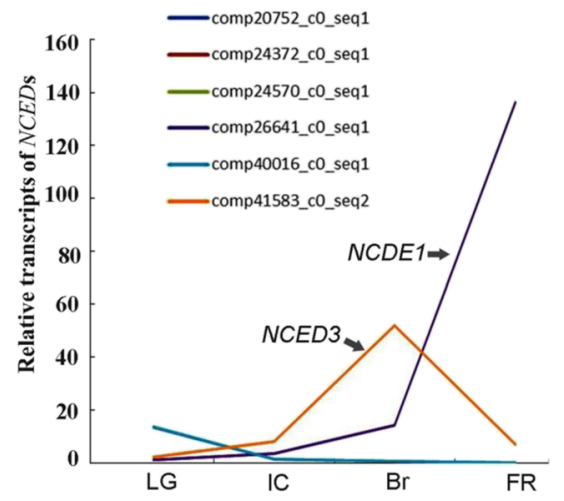

$\mathbf{E}$

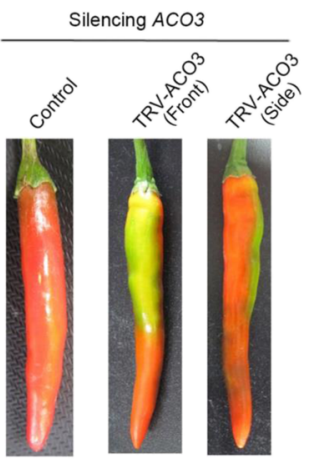

F

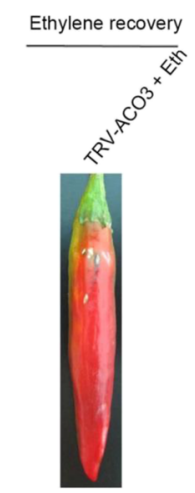

Fig. 5 Phenotypes of NCEDs-VIGS and ACO3-VIGS transgenic fruits. Four ACO gene family members (a) and three NCED1/3 gene family members (b) were identified based on the $\log _{2}$ gene expression levels obtained from RNA-seq data during fruit ripening, including the $L G, I C, B r$, and $F R$ stages. (c) Nineteen-day-old LG fruit attached to the plants were used for VIGS. (d) About 1 week after infiltration, the NCED1/3-VIGS fruits exhibited colour development, while the control fruits infiltrated with TRV alone remained in the LG stage. (e) Three weeks after infiltration, the ACO3-VIGS fruits exhibited a chimeric phenotype, while the control fruits infiltrated with TRV alone had reached the FR stage. (f) Exogenous $50 \mathrm{\mu M}$ ethephon rescued the chimeras, with ripening to the FR stage. (g) ACO3 mRNA expression in VIGS fruits was downregulated more than $80 \%$ relative to the control, and the average levels of NCED1 and NCED3 expression in VIGS fruits were downregulated more than $70 \%$ relative to the control. Ubi3 expression served as the internal control. Error bars represent the SE $(n=3)$. An asterisk $\left(^{*}\right)$ in the columns with same colour indicates a statistically significant difference $(P<0.05)$ based on analysis of variance followed by Duncan's multiple-range test

gene expression, carotenoid accumulation, and ripening. By contrast, the downregulation of ACO3 expression inhibited ethylene synthesis, finally inhibiting carotenoid biosynthesis-related gene expression, carotenoid accumulation, and ripening.

\section{Discussion}

During ripening, pepper (Capsicum) fruits display a range of colours, including green, yellow, orange, brown, and red, which are due to differences in carotenoid composition and amount [41, 42]. Generally, degradation of chlorophyll together with development of colour is associated with ripening [43]. Pepper fruits with both chlorophyll and red carotenoids appear brown [41]. Interestingly, both climacteric tomato fruit and non-climacteric strawberry fruit exhibit a white stage coupled with chlorophyll degradation during ripening $[25,44]$. A recent study showed that the white fruit is a distinct developmental stage and represents the onset of fruit ripening in strawberry [45]. In this study, the colouration by carotenoids in a green background resulted in a brown colour in 'Chaotianjiao 6' pepper fruit, which does not have a white stage (Fig. 1). This phenomenon suggests the presence of a ripening mechanism in hot pepper fruit that is distinct from that of tomato or strawberry fruit.

It is well accepted that climacteric fruit ripening shows peaks in ethylene production and respiration, whereas non-climacteric fruit ripening exhibits no significant 
A

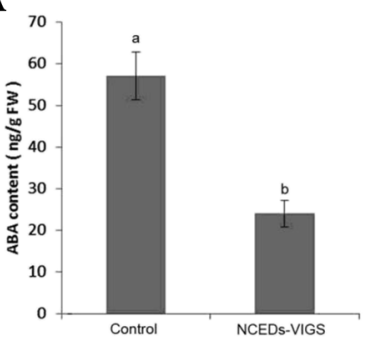

B

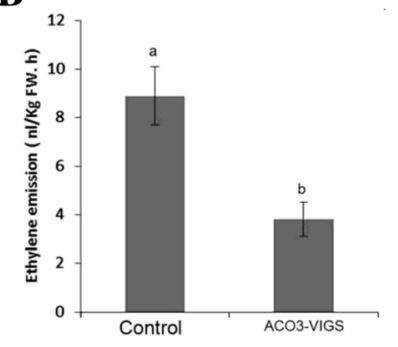

$\mathbf{C}$

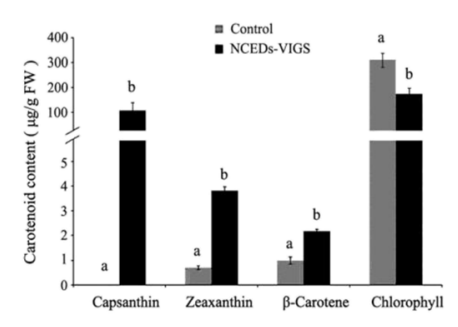

D

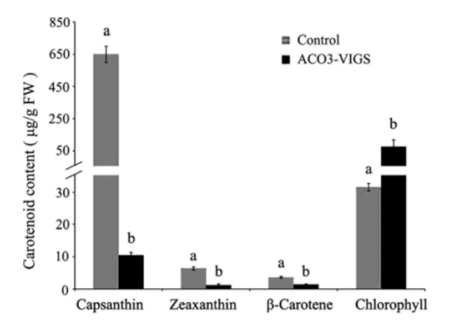

$\mathbf{E}$

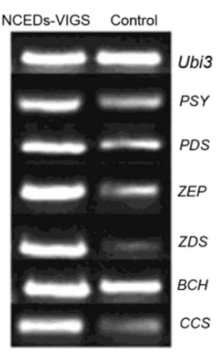

$\mathbf{F}$

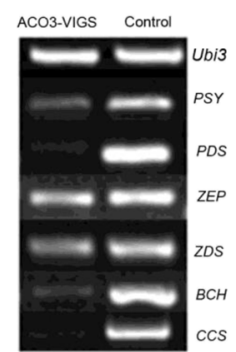

Fig. 6 Changes in several physiological and molecular parameters of transgenic and control fruits. Transgenic fruits, in which NCED and ACO3 transcripts were downregulated by more than 70 and 80\% relative to control fruits, respectively, were examined. NCEDs-VIGS represents NCED1VIGS and NCED3-VIGS mixed samples. (a) ABA contents. (b) Ethylene emission. (c) and (d) Chlorophyll and carotenoid contents. (e) and (f) Transcript levels of the PSY, PDS, ZEP, ZDS, BCH, and CCS carotenoid biosynthesis genes. PSY: 15-cis-phytoene synthase; PDS: 15-cis-phytoene desaturase; ZEP: zeaxanthin epoxidase; ZDS: zeta-carotene desaturase; BCH: beta-carotene hydroxylase 1; CCS: capsanthin/capsorubin synthase). Ubi3 was used as the internal control. Error bars represent the SE $(n=3)$. Columns with different letters $(\mathbf{a}, \mathbf{b})$ indicate statistically significant difference $(p<0.05)$ when the data are performed by variance analysis followed by Duncan's multiple range tests

increase in ethylene production or respiration [46]. Pepper fruit ripening has been widely classified as non-climacteric [35-37, 46, 47]. Nevertheless, the hot pepper fruit ripening exhibits climacteric characteristics [39]. The ripening of some peppers ('Camelot', 'King Arthur', and 'Tabasco') exhibits characteristics intermediate between climacteric and non-climacteric [48]. Interestingly, during 'Bukang' pepper fruit ripening, expression of genes involved in ethylene biosynthesis, such as $A C S$ and $A C O$, is not induced, while genes encoding ethylene signalling components, such as the EIL-like gene, are induced [49]. Although tomato and pepper belong to the Solanaceae family and accumulate carotenoids during ripening, and tomato is used as a model system of climacteric fruit ripening, pepper fruits exhibit diverse ripening types.

Capsicum frutescens fruits are generally small, hot, upright, spindle shaped, and red at the ripening stage [50]. In this study, we used the hot, upright, clustered, bullet-shaped, red pepper (C. frutescens cv. 'Chaotianjiao 6') fruit to assess the regulation of ripening by ethylene and ABA. We provide physiological and molecular evidence that ethylene and ABA regulate pepper fruit ripening differently in a process distinct from the climacteric and non-climacteric types of ripening: (1) a peak of ethylene emission was observed at the IC stage, followed by a peak in the RQ at the $\mathrm{Br}$ stage and rapid accumulation of $\mathrm{ABA}$ after the $\mathrm{Br}$ stage, which reached a maximum level at the FR stage (Fig. 1); (2) ethylene promoted ripening, ABA played no role in ripening, and fluridone accelerated ripening (Fig. 2); (3) the most abundant phytohormone-related DEGs were related to ABA (10 genes) and ethylene (6 genes), and no more than two DEGs related to other phytohormones were identified (Fig. 4); especially higher expression was observed for $A C O 3$, which is key to ethylene biosynthesis, and for NCED1/3, which are key to ABA biosynthesis (Fig. 5); and (4) the downregulated expression of NCED1/3 and $A C O 3$ promoted and inhibited colouration, respectively, consistent with the results of our physiological and molecular analyses (Figs. 5, 6).

To our surprise, exogenous ABA played no role in colouration, but fluridone did promote colouration (Fig. 2). This phenomenon was also confirmed by the downregulation of NCED1/3 expression by VIGS (Fig. 5). Similarly, a significant reduction in NCED activity leading to carotenoid accumulation was previously reported in tomato fruit [51]. Indeed, pepper fruit colours result from the composition and amount of diverse carotenoids, which are synthesised by a set of genes including PSY, PDS, ZDS, BCH, $Z E P$, and $C C S[40,42,52,53]$, consistent with our RNA-seq data (Fig. 4). To some extent, the ZEP/ABA1 screen is a common substrate for carotenoids and ABA 
biosynthesis, which may reflect a complex role for $\mathrm{ABA}$ in hot pepper fruit ripening.

Two early, sequential committed steps in the conversion of farnesyl pyrophosphate (FPP) to phytoene in carotenoid biosynthesis are catalysed by geranylgeranyl diphosphate synthase and phytoene synthases [54]. Notably, the recent reports show that squalene synthase (SQS) is involved in carotenoid biosynthesis, and can convert to a dehydrosqualene synthase for production of the $\mathrm{C}_{30}$ carotenoid backbone $[55,56]$. An early report showed the conversion of FPP to squalene by SQS in hot pepper [57]. In this study, the transcriptome data showed that $S Q S$ expression levels, on the whole, exhibited a continually decreasing trend at the onset of ripening (Additional file 1: Figure S2a). Fluridone can inhibit PDS activity [58, 59]. We showed that fluridone led to an increase in $S Q S$ transcript levels in fluridone-treated fruits (Additional file 1: Figure S2b), finally initiated the 'squalene route' for carotenoid biosynthesis [56]. To a large extent, the colouration of pepper fruit promoted by fluridone treatment resulted from the upstream accumulation of $\mathrm{C}_{30}$ carotenoid once PDS activity is inhibited by fluridone.

Paradoxically, although endogenous ABA levels increased rapidly after the $\mathrm{Br}$ stage (Fig. 1), exogenous ABA did not affect colouration (Fig. 2). Concurrent with ABA accumulation during colouration, the transcript levels of $S n R K 2$, a positive regulator of ABA signalling in the ABA'PYR-PP2C-SnRK2' core signalling pathway [26], declined continuously, while the transcript levels of $P P 2 C$, a negative regulator of ABA signalling, increased continuously (Figs. 1,4) and inhibited ABA signalling at the transcriptional level. In contrast, concurrent with a decrease in ethylene emission during colouration, the expression of ethylene signalling-related DEGs, including CTR1, CTR2, EIN2, EIN3, and ERF1, promoted ethylene signalling (Figs. 1, 4). In conclusion, a peak in ethylene emission resulting from high $A C O 3$ mRNA expression levels at the IC stage triggered the hot pepper fruit ripening through a coordinated expression pattern of ethylene-signalling genes during colouration (Figs. 1, 4). Although a rapid increase in ABA content was consistent with high NCED1 mRNA expression levels during fruit colouration, ABA signalling was weakened by the uncoordinated expression of ABA-signalling genes (Figs. 1, 4). Ethylene and $A B A$ regulate hot pepper fruit ripening differently, with ethylene promotes colouration while ABA might play a role in de-greening. Our results provide new insights into understanding of fleshy fruit ripening.

\section{Conclusions}

We have provided a series of pharmacological, physiological and molecular evidences to demonstrate that during hot pepper (C. frutescens) fruit ripening, higher
ACO3 expression levels are associated with an ethylene emission peak, which triggers the onset of ripening, demonstrating that ethylene acts as a positive regulator of the ripening. The higher NCED1/3 expression levels are coupled with ABA accumulation during ripening. Exogenous $\mathrm{ABA}$ does not promote colouration, and inhibition of ABA biosynthesis accelerates colouration. Our findings provide new insights into processes of fleshy fruit ripening regulated by $\mathrm{ABA}$ and ethylene, focusing on ethylene in carotenoid biosynthesis and $\mathrm{ABA}$ in chlorophyll degradation.

\section{Methods}

\section{Plant material}

Red, clustered, upright, hot pepper (C. frutescens 'Chaotianjiao 6') plants were grown in a greenhouse $\left(25-35{ }^{\circ} \mathrm{C}\right.$, relative humidity $70-90 \%, 12 / 12$-h light/dark regime) in 2015 and 2016. Four hundred flowers on 50 plants were tagged during flowering. Developmental fruits were divided into six stages (YG, SG, LG, IC, Br, and FR) at $1,2,3,4,5$, and 6 weeks after anthesis, respectively.

\section{Determination of ethylene production}

Ten uniform pepper fruits attached to the plants at each stage were sampled and placed in 1-L glass jars for $1 \mathrm{~h}$ at $25{ }^{\circ} \mathrm{C}$. One millilitre of the jar headspace gas was extracted and injected into a gas chromatograph equipped with a flame ionisation detector and an activated alumina column (model $6890 \mathrm{~N}$; Agilent Technologies, USA). Ethylene production ( $\left.\mu \mathrm{L} \mathrm{hr} .^{-1} \mathrm{~kg}^{-1} \mathrm{FW}\right)$ was analysed by column chromatography, including $5 \%$ phenyl methyl siloxane and a 30-m capillary alumina column (19,091 J-413; Agilent Technologies). The temperatures of the column and detector were $80{ }^{\circ} \mathrm{C}$ and $150{ }^{\circ} \mathrm{C}$, respectively. The rate of $\mathrm{N}^{2}$ carrier gas flow was $40 \mathrm{~mL} \mathrm{~min}^{-1}$, and the hydrogen pressure was $0.6 \mathrm{~kg} \mathrm{~cm}^{-2}$. The experiment was repeated three times.

\section{Determination of ABA content}

Three frozen pepper fruits of each ripening stage were selected randomly for determination of ABA content by gas chromatography-mass spectroscopy (GC-MS). One gram of pericarp tissue was mixed with antioxidant copper reagent, quartz sand, a small amount of $80 \%$ cold methanol, and $\mathrm{D}_{3}$-ABA (an internal standard). The mixture was ground and mixed with $80 \%$ methanol, then soaked overnight at $4{ }^{\circ} \mathrm{C}$ for $12 \mathrm{~h}$. Filtration, supernatant collection, extraction with a $\mathrm{C}_{18}$ column, and GC-MS analysis were performed as described previously [25]. The experiment was repeated three times.

\section{Determination of carotenoid and chlorophyll contents}

Three uniform pepper fruits of each ripening stage were used to determine carotenoid and chlorophyll contents 
by high-performance liquid chromatography (HPLC) using $5 \mathrm{~g}$ pericarp tissue. $ß$-Carotene, zeaxanthin, and capsanthin were purchased from Sigma-Aldrich (Munich, Germany). A Sep-Pak Silica 6-cc Vac Cartridge solid-phase extraction column (Waters, Milford, MA, USA) was used. Acetonitrile, methanol, and methyl tert-butyl ether were HPLC grade. Chromatographic analysis was performed using an Agilent 1260 HPLC system. An analytical C30 column $(250 \times 4.6 \mathrm{~mm}$, $5 \mu \mathrm{m}$; YMC Co., Japan) was used. Separation was carried out using methanol/tert-butyl methyl ether $(70: 30, v / \mathrm{v})$ at a flow rate of $1.0 \mathrm{~mL} / \mathrm{min}$ with a column temperature of $25^{\circ} \mathrm{C}$. Chromatograms were monitored at a UV wavelength of $450 \mathrm{~nm}$. The procedures were carried out as described previously [40]. Chlorophyll contents were determined as described previously [60]. The experiments were repeated three times.

\section{Determination of soluble sugar and starch contents}

Three uniform pepper fruits of each ripening stage were used to determine the sugar contents of pericarp tissue (1 g) using reverse-phase HPLC (RID1 A detector, 1200 series; Agilent Technologies). The supernatant was fractionated using a Sugar-PakTM1 column $(6.5 \times 300 \mathrm{~mm}$, Waters) with $100 \%$ MilliQ water for $25 \mathrm{~min}$ at the flow rate of $0.4 \mathrm{~mL}$ per min. The column temperature was $80^{\circ} \mathrm{C}$ and the injection volume was $20 \mu \mathrm{L}$. Standard samples used were $\mathrm{D}-(+)$ glucose, $\mathrm{D}-(-)$ fructose, and sucrose (Sigma-Aldrich). The HPLC analysis was done as described previously [25]. Starch contents were detected using a total starch assay kit (Megazyme International Ireland Ltd., Wicklow, Ireland) according to the manufacturer's protocol. The experiments were repeated three times.

\section{Determination of RQ}

Three uniform pepper fruits of each ripening stage were used to determine the RQ of pericarp tissue $(5 \mathrm{~g})$ using a Warburg microrespirometer. Absorption of oxygen was determined with the addition of $20 \% \mathrm{NaOH}$ solution to the reaction bottle, and the release of carbon dioxide was determined with the removal of $20 \% \mathrm{NaOH}$ solution. The RQ was the ratio of the amount of carbon dioxide released to the amount of oxygen absorbed. The experiment was repeated three times.

\section{Effects of ABA, ethylene, and inhibitors on pepper fruit ripening}

Seven LG fruits attached to plants were selected and immersed for $2 \mathrm{~min}$ in $50 \mu \mathrm{M}$ ABA, $100 \mu \mathrm{M}$ fluridone, $50 \mu \mathrm{M}$ ethephon (2-chloroethylphosphonic acid, an ethylene-releasing agent), or $1 \mathrm{~mL} / \mathrm{L} 1-\mathrm{MCP}$ (an inhibitor of ethylene release), as described previously [61, 62], with immersion in water used as a control. The treated and control fruits were placed in a growth chamber $\left(25^{\circ} \mathrm{C}\right.$, relative humidity $70-80 \%, 12 / 12$-h light/dark regime) for 8 days. The experiment was repeated three times.

\section{RNA extraction, library construction, and RNA-seq analysis}

RNAs were extracted from pericarp tissues from four uniform pepper fruits of each ripening stage, including LG, IC, Br, and FR, using the RNeasy Plant Mini Kit (Qiagen, Dusseldorf, Germany) for RNA-seq analysis. DNase digestion by RNase-Free DNase (Qiagen) removed contaminating DNA, and RNA samples were processed sequentially using an RNA Library Prep Kit (New England BioLabs, Ipswich, MA, USA). RNA-seq and data analysis were performed on the Illumina HiSeq2000 (Illumina, San Diego, CA, USA) by the Beijing Yuanyi Gene Science Company (Beijing, China). Unigenes with adjusted $P$-values $<0.05$ and absolute values of $\log _{2}$ (expression fold change) $>1$ were deemed to be differentially expressed, and false discovery rate-adjusted $P$-values $<0.05$ for unigenes were considered to be statistically significant [63-67]. The experiment was repeated three times.

\section{VIGS}

The pTRV1 and pTRV2 vectors were kindly provided by Dr. Yu-le Liu (Qinghua University, Beijing, China). On the basis of the RNA-seq data, primers with an XbaI restriction site in the forward primer and a $K p n 1$ restriction site in the reverse primer (underlined below) were designed for amplification of the partial gene sequences of ACO3 (GenBank accession no. NM_001324767), NCED1 (GenBank accession no. XM_016697078), and NCED3 (GenBank accession no. XM_016691094). The primers used were as follows: a 452-bp cDNA fragment of ACO3 (forward, 5'-GCTCTAGAAAAAGGGCTGA AACAATGGAC-3' and reverse, 5'-GGGGTACCGGAC AAGGAGGGTAG TTGCTAA-3'), a 537-bp cDNA fragment of NCED1 (forward, 5'-GCTCTAGAAATCTCTAC AAGTGACCGGA-3' and reverse, 5'-GGGGTACCGAAT CACATCATAGCTAAGAG-3'), and a 600-bp cDNA fragment of NCED3 (forward, 5'-GCTCTAGACAGTGAGTT ATTCTTGCCGTT-3' and reverse, 5'-GGGGTACCACCT GGATTTCTTCTTTTTG TC-3'). These amplified fragments were inserted into the pEASY-T1 Simple Cloning Kit (TransGen Biotech, Beijing, China), and then into the TRV pTRV2 vector between the XbaI and Kpn1 sites. The Agrobacterium tumefaciens strain GV3101 containing pTRV1, pTRV2, and pTRV2-ACO3/NCED1/NCED3 was used for VIGS. Cell suspension of each TRV2 vector carrying target genes was mixed with TRV1 cell suspension at a 1:1 $(v / v)$ ratio, and $0.2-\mathrm{mL}$ volumes were infiltrated into seven LG fruits as described previously [40]. Empty 
TRV vector was used as a control. After inoculation, the fruits were covered with black paper for $24 \mathrm{~h}$. The experiment was repeated three times.

\section{qPCR}

The relative expression levels of carotenoid synthesis genes in transgenic fruits were determined using semi-quantitative reverse-transcription PCR. Three transgenic fruits were sampled for RNA isolation and cDNA synthesis as described above using pericarp tissue. First-strand cDNAs corresponding to the genes were used as templates during PCR amplification through 24 or 28 cycles. Expression of the Ubi3 gene was used as an internal reference [40]. The primers for qPCR are shown in Additional file 1: Table S1. The experiment was performed with three replications.

\section{Additional files}

Additional file 1: Table S1. The primers for qPCR based on RNA-Seq data. Figure S1. Phenotype of the red, cluster, upright and hot pepper plant (Capsicum frutescens, cv. 'Chaotianjiao 6'). Figure S2. Fluridonepromotion of the fruit coloration is related to squalene synthase (SQS) expression at transcription levels. (DOC $4362 \mathrm{~kb}$ )

\section{Abbreviations}

AAO: Abscisic-aldehyde oxidase; ABA: Abscisic acd; ABI: Abscisic acidinsensitive; ACO: 1-aminocyclopropane-1-carboxylate oxidase; ARR: Twocomponent response regulator; $\mathrm{BCH} / \mathrm{crtZ}$ : Beta-carotene hydroxylase; CCS/ IcyB/crtY: Capsanthin/capsorubin synthase; CTR: Ethylene response sensor; CYP707: Abscisic acid 8'-hydroxylase; EIN: Ethylene-insensitive protein; ERF: Ethylene-responsive transcription factor; GH3: Indole-3-acetic acid-amido synthetase; GID1: Gibberellin receptor; IAA: Auxin-responsive protein; MYC2: Myelocytomatosis transcription factor; NCED: 9-cis-epoxycarotenoid dioxygenase; PDS/crtP: 15-cis-phytoene desaturase; PP2C: Protein phosphatase 2C; PSY/crtB: 15-cis-phytoene synthase; PYL: Abscisic acid receptor; SnRK: Serine/threonine-protein kinase; ZDS/crtQ: Zeta-carotene desaturase; ZEP/ABA: Zeaxanthin epoxidase

\section{Acknowledgments}

We thank Dr. Yule Liu for providing TRV1 and TRV2 vector.

\section{Funding}

This work was supported the National Natural Science Foundation of China (Project 31672125), the Natural Science Foundation of Beijing Municipality (6171001), Beijing Training Project for the Leading Talents in S \& T (grant No. LJ201612), Sichuan Lomon Biotechnology Co., LTD (2018001).

\section{Authors' contributions}

HBZ was involved in data interpretation. LCL performed the experiments. HYY cultivated the pepper plants. SYY designed the research and wrote the article. All authors read and approved the final version of the manuscript.

\section{Ethics approval and consent to participate}

Not applicable.

\section{Consent to publication}

Not applicable.

\section{Competing interests}

The authors declare that they have no competing interests

\section{Publisher's Note}

Springer Nature remains neutral with regard to jurisdictional claims in published maps and institutional affiliations.

Received: 7 November 2017 Accepted: 30 July 2018

Published online: 10 August 2018

\section{References}

1. Howard LR, Talcott ST, Brenes CH, Villalon B. Changes in phytochemical and antioxidant activity of selected pepper cultivars (Capsicum species) as influenced by maturity. J Agric Food Chem. 2000;48:1713-20.

2. Smith PG, Villalon B, Villa PL. Horticultural classification of peppers grown in the United States. HortSci. 1987:22:11-3.

3. Dias GB, Gomes VM, Moraes TM, Zottich UP, Rabelo GR, Carvalho AO, Moulin M. Characterization of Capsicum species using anatomical and molecular data. Genet Mol Res. 2013;12:6488-501.

4. Moses M, Umaharan P, Dayanandan S. Microsatellite based analysis of the genetic structure and diversity of Capsicum chinense in the Neotropics. Genet Resour Crop Evol. 2014;61:741-55.

5. Giovannoni JJ. Genetic regulation of fruit development and ripening. Plant Cell. 2004;16:170-80

6. Klie S, Osorio S, Tohge T, Drincovich MF, Fait A, Giovannoni JJ, Fernie AR. Conserved changes in the dynamics of metabolic processes during fruit development and ripening across species. Plant Physiol. 2014;164:55-68.

7. Palma JM, Corpas FJ, Del Río LA, López-Huertas E. Pepper fruit as a model to study the metabolism of antioxidants, ROS and RNS. Free Radic. Biol. Med. 2014;75:S39.

8. Villavicencio L, Blankenship S, Sanders D, Swallow W. Ethylene and carbon dioxide production in detached fruit of selected pepper cultivars. J Am Soc Hortic Sci. 1999:124:402-6.

9. Brady CJ. Fruit ripening. Annu Rev Plant Biol. 1987;38:155-78.

10. Lelièvre $J M$, Latchè $A$, Jones $B$, Bouzayen $M$, Pech JC. Ethylene and fruit ripening. Physiol Plant. 1997;101:727-39.

11. Giovannoni J. Molecular biology of fruit maturation and ripening. Annu. Rev. plant Physiol. \& plant Mol. Biol. 2001;52:725-49.

12. Iqbal N, Khan NA, Ferrante A, Trivellini A, Francini A, Khan MIR. Ethylene role in plant growth, development and senescence: interaction with other phytohormones. Front Plant Sci. 2017;8:475.

13. Alexander $L$, Grierson D. Ethylene biosynthesis and action in tomato: a model for climacteric fruit ripening. J Exp Bot. 2002;53:2039-55.

14. Cherian S, Figueroa CR, Nair H. 'Movers and shakers' in the regulation of fruit ripening: a cross-dissection of climacteric versus non-climacteric fruit. J Exp Bot. 2014;65:4705-22.

15. Yang SF, Hoffman NE. Ethylene biosynthesis and its regulation in higher plants. Annu Rev Plant Physiol. 1984;35:155-89.

16. Klee HJ, Giovannoni JJ. Genetics and control of tomato fruit ripening and quality attributes. Annu Rev Genet. 2011:45:41-59.

17. Seymour GB, Østergaard L, Chapman NH, Knapp S, Martin C. Fruit development and ripening. Annu Rev Plant Biol. 2013;64:219-41.

18. Pesaresi P, Mizzotti L, Colombo M, Masiero S. Genetic regulation and structural changes during tomato fruit development and ripening. Front Plant Sci. 2014:5:124.

19. Liu M, Pirrello J, Chervin C, Roustan JP, Bouzayen M. Ethylene control of fruit ripening: revisiting the complex network of transcriptional regulation. Plant Physiol. 2015;169:2380-90.

20. Giovannoni J, Nguyen C, Ampofo B, Zhong S, Fei Z. The epigenome and transcriptional dynamics of fruit ripening. Annu Rev Plant Biol. 2017;68:61-84.

21. Vrebalov J, Ruezinsky D, Padmanabhan V, White R, Medrano D, Drake R, Schuch W. A MADS-box gene necessary for fruit ripening at the tomato ripening-inhibitor (rin) locus. Science. 2002;296:343-6.

22. Chai YM, Jia HF, Li CL, Dong QH, Shen YY. FaPYR1 is involved in strawberry fruit ripening. J Exp Bot. 2011;62:5079-89.

23. Li CL, Jia HF, Chai YM, Shen YY. Abscisic acid perception and signaling transduction in strawberry: a model for non-climacteric fruit ripening. Plant Signal Behav. 2011;6:1950-3.

24. Li Q, Ji K, Sun Y, Luo H, Wang H, Leng P. The role of FaBG3 in fruit ripening and B. cinerea fungal infection of strawberry. Plant J. 2013;76:24-35.

25. Jia HF, Chai YM, Li CL, Lu D, Luo JJ, Qin L, Shen YY. Abscisic acid plays an important role in the regulation of strawberry fruit ripening. Plant Physiol. 2011;157:188-99. 
26. Jia HF, Lu D, Sun JH, Li CL, Xing Y, Qin L, Shen YY. Type $2 C$ protein phosphatase $A B \mid 1$ is a negative regulator of strawberry fruit ripening. J Exp Bot. 2013;64:1677-87.

27. Han Y, Dang R, Li J, Jiang J, Zhang N, Jia M, Wei L, et al. SUCROSE NONFERMENTING1-RELATED PROTEIN KINASE2.6, an ortholog of OPEN STOMATA1, is a negative regulator of strawberry fruit development and ripening. Plant Physiol. 2015;167:915-30.

28. Kadomura-Ishikawa Y, Miyawaki K, Takahashi A, Masuda T, Noji S. Light and abscisic acid independently regulated FaMYB10 in Fragaria $\times$ ananassa fruit. Planta. 2015:241:953-65.

29. Medina-Puche L, Blanco-Portales R, Molina-Hidalgo FJ, Cumplido-Laso G, García-Caparrós N, Moyano-Cañete E, Caballero-Repullo JL. Extensive transcriptomic studies on the roles played by abscisic acid and auxins in the development and ripening of strawberry fruits. Funct. Integr. Genomics. 2016:16:671-92

30. Shen YY, Rose JKC. ABA metabolism and signaling in fleshy fruits. In: Zhang DP, editor. Abscisic Acid: Metabolism, Transport and Signaling. Dordrecht: Springer Science + Business Media; 2014. p. 271-86.

31. Leng P, Yuan B, Guo Y. The role of abscisic acid in fruit ripening and responses to abiotic stress. J Exp Bot. 2014;65:4577-88.

32. Obando J, Miranda C, Jowkar MM, Moreno E, Souri MK, Martinez IA, Arus P. Creating climacteric melon fruit from non-climacteric parentals: postharvest quality implications. In: Ramina A, Chang J, Giovannoni J, Klee H, Perata P, Woltering $E$, editors. Advances in plant ethylene research. Proceedings of the 7th international symposium on plant hormone ethylene. Dordrecht: Kluwer; 2007. p. 197-205.

33. Paul V, Pandey R, Srivastava GC. The fading distinctions between classical patterns of ripening in climacteric and non-climacteric fruit and the ubiquity of ethylene-an overview. J Food Sci Technol. 2012;49:1-21.

34. Saltveit ME. Carbon dioxide, ethylene and color development in ripening mature green bell peppers. J Am Soc Hortic Sci. 1977;102:523-5.

35. Lurie S, Shapiro B, Ben-Yehoshua S. Effects of water stress and degree of ripeness on rate of senescence of harvested bell pepper fruit. J Am Soc Hortic Sci. 1986;111:880-5.

36. Lu G, Yang C, Liag H, Lu Z. 'Changjiao' hot peppers are non-climacteric. HortSci. 1990:25:807.

37. Biles $C L$, Wall MM, Blackstone K. Morphological and physiological changes during maturation of new Mexican type peppers. J Soc Hort Sci. 1993;118: 476-80

38. Aizat WM, Able JA, Stangoulis JC, Able AJ. Characterisation of ethylene pathway components in non-climacteric capsicum. BMC Plant Biol. 2013:13:191.

39. Gross KC, Watada AE, Kang MS, Kim SD, Lee SW. Biochemical changes associated with the ripening of hot pepper fruit. Physiol Plant. 1986;66:31-6.

40. Tian SL, Li L, Chai WG, Shah SN, Gong ZH. Effects of silencing key genes in the capsanthin biosynthetic pathway on fruit color of detached pepper fruits. BMC Plant Biol. 2014;14:314.

41. Borovsky $Y$, Paran I. Chlorophyll breakdown during pepper fruit ripening in the chlorophyll retainer mutation is impaired at the homolog of the senescence-inducible stay-green gene. Theor Appl Genet. 2008;117:235-40.

42. Kim OR, Cho MC, Kim BD, Huh JH. A splicing mutation in the gene encoding phytoene synthase causes orange coloration in Habanero pepper fruits. Mol Cells. 2010:30:569-74.

43. Pratt HK, Goeschl JD. Physiological roles of ethylene in plants. Annu Rev Plant Physiol. 1969;20:541-8.

44. Mes PJ, Boches P, Myers JR. Characterization of tomatoes expressing anthocyanin in the fruit. J Am Soc Hortic Sci. 2008;133:262-9.

45. Wang QH, Zhao C, Zhang M, Li YZ, Shen YY, Guo JX. Transcriptome analysis around the onset of strawberry fruit ripening uncovers an important role of oxidative phosphorylation in ripening. Sci Rep. 2017;7:41477.

46. Biale JB. Growth, maturation, and senescence in fruit. Science. 1964;146: 880-8.

47. Miller $\mathrm{CH}$, McCollum RE, Claimon S. Relationship between growth of bell peppers (Capsicum annuum L.) and nutrient accumulation during ontogeny in field environments. J. Amer. Soc. Hort. Sci. 1979;104:852-7.

48. Villavicencio LE, Blankenship SM, Sanders DC, Swallow WH. Ethylene and carbon dioxide concentrations in attached fruits of pepper cultivars during ripening. Sci Hort. 2001;91:17-24.

49. Lee $S$, Chung EJ, Joung $Y H$, Choi D. Non-climacteric fruit ripening in pepper: increased transcription of ElL-like genes normally regulated by ethylene. Funct Integr Genomics. 2010;10:135-46.
50. Carvalho SI, Ragassi CF, Oliveira IB, Amaral ZP, Reifschneider FJ, Faleiro FG, Buso GS. Transferability of microsatellite markers of Capsicum annuum L. to C. Frutescens L. and C. Chinense Jacq. Genet. Mol. Res. 2015;14:7937-46.

51. Sun L, Yuan B, Zhang M, Wang L, Cui M, Wang Q, Leng P. Fruit-specific RNAi-mediated suppression of SINCED1 increases both lycopene and $\beta$ carotene contents in tomato fruit. J Exp Bot. 2012;63:3097-108.

52. Lefebvre V, Kuntz M, Camara B, Palloix A. The capsanthin-capsorubin synthase gene: a candidate gene for the $y$ locus controlling the red fruit colour in pepper. Plant Mol Biol. 1998;36:785-9.

53. Martínez-López LA, Ochoa-Alejo N, Martínez O. Dynamics of the chili pepper transcriptome during fruit development. BMC Genomics. 2014; 15:143

54. Von L, Welsch R, Bonk M, Giuliano G, Batschaue A, Kleinig H. Lightdependent regulation of carotenoid biosynthesis occurs at the level of phytoene synthase expression and is mediated by phytochrome in Sinapis alba and Arabidopsis thaliana seedlings. Plant J. 1997;12:625-34.

55. Furubayashi M, Li L, Katabami A, Saito K, Umeno D. Directed evolution of squalene synthase for dehydrosqualene biosynthesis. FEBS Lett. 2014;588: 3375-81.

56. Furubayashi M, Li L, Katabami A, Saito K, Umeno D. Construction of carotenoid biosynthetic pathways using squalene synthase. FEBS Lett. 2014; 588:436-42.

57. Lee JH, Yoon YH, Kim HY, Shin DH, Kim DU, Lee IJ, Kim KU. Cloning and expression of squalene synthase cDNA from hot pepper (Capsicum annuum). Mol. Cells. 2002;13:436-43.

58. Steiger S, Perez-Fons L, Fraser PD, Sandmann G. Biosynthesis of a novel C30 carotenoid in Bacillus firmus isolates. J Appl Microbiol. 2012;113:888-95.

59. Vaisberg AJ, Schiff JA. Events surrounding the early development of Euglena chloroplasts. Plant Physiol. 1975;57:260-9.

60. Doong RL, MacDonald GE, Shilling DG. Effect of fluridone on chlorophyll, carotenoids, and anthocyanin content of hydrilla. J Aquat Plant Manag. 1993:31:55-9.

61. Zhang $M$, Yuan $B$, Leng $P$. The role of $A B A$ in triggering ethylene biosynthesis and ripening of tomato fruit. J Exp Bot. 2009;60:1579-88.

62. Villarreal NM, Bustamante CA, Civello PM. Effect of ethylene and 1-MCP treatments on strawberry fruit ripening. J Sci Food Agric. 2010;90:683-9.

63. Mortazavi A, Williams BA, McCue K, Schaeffer L, Wold B. Mapping and quantifying mammalian transcriptomes by RNA-Seq. Nat Methods. 2008;5: $621-8$.

64. Benjamini $Y$, Yekutieli $D$. The control of the false discovery rate in multiple testing under dependency. Ann Stat. 2001;29:1165-88.

65. Wang Z, Fang B, Chen J, Zhang X, Luo Z, Huang L, Chen X. De novo assembly and characterization of root transcriptome using Illumina pairedend sequencing and development of SSR markers in sweet potato (Ipomoea batatas). BMC Genomics. 2010;11:726.

66. Langmead B, Salzberg SL. Fast gapped-read alignment with bowtie 2. Nat mathods. 2012;9:357-9.

67. Ming YL, Gui RQ, Jing J, Hui QY, Li HX, Jin ZX, Ren YZ. Transcriptome sequencing and de novo analysis for bamboo using the illumina platform. PLOS One. 2012:7:1-11.

Ready to submit your research? Choose BMC and benefit from:

- fast, convenient online submission

- thorough peer review by experienced researchers in your field

- rapid publication on acceptance

- support for research data, including large and complex data types

- gold Open Access which fosters wider collaboration and increased citations

- maximum visibility for your research: over $100 \mathrm{M}$ website views per year

At BMC, research is always in progress.

Learn more biomedcentral.com/submission 